\title{
Detection of EGFR- and HER2-activating mutations in squamous cell carcinoma involving the head and neck
}

\author{
Carlynn Willmore-Payne, Joseph A Holden and Lester J Layfield \\ Department of Pathology, University of Utah Health Sciences Center, Salt Lake City, UT, USA
}

\begin{abstract}
Recent reports have indicated that mutations in the epidermal growth factor receptor-1 (EGFR) occur in about $7 \%$ of patients with squamous cell carcinoma of the head and neck. It is known that many patients with nonsmall-cell lung cancer who respond to the EGFR inhibitors gefitinib and erlotinib have tumors with EGFRactivating mutations. This might suggest that patients with head and neck squamous carcinoma, who also have tumors with EGFR-activating mutations, might represent a patient population who could benefit from gefitinib or erlotinib therapy. High-resolution melting amplicon analysis (HRMAA) is a recently described technique which can be used for screening tumor DNA isolated from paraffin blocks for tyrosine kinase-activating mutations. In this report we screened 24 cases of squamous cell carcinoma, either primary in the head and neck or secondarily involving the head and neck area, for activating mutations in EGFR exons 18, 19, 20, 21, and for HER2 exons 19 and 20. All cases were followed up by direct DNA sequencing. Two (8\%) of the 24 cases were positive. One case was a maxillary sinus tumor which contained an exon 20 -activating mutation (N771YinsG). Surprisingly, the other case was a primary squamous cell carcinoma of the skin which invaded the head and neck area only secondarily. This tumor contained an exon 19-activating mutation (G729E). No HER2-activating mutations were found. The presence of a small number of squamous cell carcinomas in the head and neck area with EGFR-activating mutations suggests the existence of a population of patients who could derive benefit from gefitinib or erlotinib therapy. HRMAA could serve as a screening method to easily and rapidly identify these patients.

Modern Pathology (2006) 19, 634-640. doi:10.1038/modpathol.3800552; published online 17 March 2006
\end{abstract}

Keywords: head and neck squamous cell carcinoma; EGFR-activating mutations; HER2-activating mutations; EGFR immunohistochemical staining

One of the exciting recent developments in clinical oncology is the realization that several classes of human malignancies are characterized by mutationally activated proteins and that these constitutively activated proteins may be solely responsible for tumor growth and survival. ${ }^{1}$ These proteins are in the tyrosine kinase class of enzymes and can be activated by a number of mechanisms. One of the most common mechanisms of activation is by somatic mutation in the tumor which activates c-kit in gastrointestinal stromal tumors (GISTs), ${ }^{2}$ BRAF in melanomas ${ }^{3}$ and papillary thyroid cancer, ${ }^{4}$ and epidermal growth factor receptor-1 (EGFR-1) ${ }^{5}$ and epidermal growth factor receptor-2 (EGFR-2,

Correspondence: Dr JA Holden, MD, PhD, Department of Pathology, University of Utah Health Sciences Center, 50 North Medical Drive, Salt Lake City, UT 84108, USA.

E-mail: joe.holden@path.utah.edu

Received 7 October 2005; revised 23 November 2005; accepted 1 December 2005; published online 17 March 2006
HER2) in lung carcinoma. ${ }^{6}$ Tumors whose growth is dependent on the constitutive signaling of these activated proteins become sensitive to small molecule tyrosine kinase active site inhibitors which 'target' the mutationally activated proteins. The remarkable success of imatinib (Gleevec), in the treatment GISTs is an example of this type of 'targeted' therapy.

Gefitinib and erlotinib are active site tyrosine kinase inhibitors of EGFR. Increased EGFR expression has been observed in many human tumor systems and therefore drugs which inhibit EGFR would seem to be a rational choice of anticancer agents. In lung cancer, recent data suggest that in many instances, patient response to EGFR-targeted anticancer therapy can be directly correlated with an underlying somatic-activating mutation in the tumor EGFR gene. The most common EGFR mutations include the deletion of four conserved aminoacid residues (LREA) in exon 19 and a point mutation, L858R in exon $21 .^{7}$ 
Current postsurgical therapy for squamous cell carcinoma of the head and neck involves radiation and chemoradiation. ${ }^{8}$ Gefitinib has been shown to have single-agent activity in head and neck squamous cell carcinoma ${ }^{9}$ but correlations with EGFRactivating mutations were not carried out because this trial was completed before it was realized that EGFR-activating mutations may be responsible for the therapeutic response. A more recent report indicates that gefitinib in combination with celecoxib has activity in advanced squamous cell carcinoma of the head and neck. ${ }^{10}$ Activating mutations in EGFR were tested for and not found, but the study was only a Phase I trial in 19 patients, not all of whom had material available for molecular testing.

Recently, a single report has appeared indicating that about $7 \%$ of head and neck squamous cell carcinomas have EGFR-activating mutations. ${ }^{11}$ Interestingly they were all the same deletion mutation in exon 19 (delE746-A750). By analogy with lung cancer, it might be predicted that head and neck squamous cell carcinoma patients with EGFRactivating mutations might represent a subset of patients who might respond to small molecule EGFR inhibitors.

In this report we used high-resolution melting amplicon analysis (HRMAA) to screen for EGFR and HER2-activating mutations in squamous cell carcinomas originating in or affecting the head and neck from 24 patients. We uncovered two EGFR mutation-positive cases (8\%). One case contained an activating mutation in exon 19 and the other was in exon 20. No HER2 mutations were found.

\section{Materials and methods}

\section{Sources of Tissue}

A total of 24 cases of squamous cell carcinoma originating in or affecting the head and neck were retrieved from the surgical pathology archives at the University of Utah Hospital. Selection of cases was based on a sufficient amount of material available for mutation analysis. All histologic sections and any accompanying immunohistochemical (IHC) stains were reviewed to confirm the diagnosis. All cases were given a number and 'anonymized' to protect patient privacy. This study has been approved by the Institutional Review Board at the University Utah (IRB\# 11903).

\section{Antibodies and IHC Staining}

IHC staining for EGFR was performed with the DAKO (Carpinteria, CA, USA) pharmDX kit for EGFR in accord with the manufacturer's instructions. Antigen retrieval was used and the immunostaining performed with the DAKO Autostainer (Carpinteria, CA, USA) in accord with the manu- facturer's instructions. The chromogen was diaminobenzidine. Interpretation of the EGFR immunostain was carried out on a 0 to $3+$ scale as described. ${ }^{12}$

\section{Chemicals, DNA, and Enzymes}

These reagents have been described previously. ${ }^{3}$

\section{Isolation of DNA from Paraffin-Embedded Tumor Tissue}

These procedures have been previously described in detail. ${ }^{3}$ Briefly, after reviewing the hematoxylin and eosin $(\mathrm{H}$ and $\mathrm{E})$-stained slides, the corresponding paraffin block containing sufficient tumor tissue was selected. Only areas in which it was estimated that there was at least $50 \%$ tumor were analyzed. The area of squamous cell carcinoma on the $\mathrm{H}$ and $\mathrm{E}$ slide was outlined with a felt tip pen and the corresponding area in an unstained slide was microdissected with a scalpel. The microdissected tissue was digested in a Tris buffer containing Tween 20 and proteinase $\mathrm{K}$. After boiling for $10 \mathrm{~min}$ to inactivate the proteinase $\mathrm{K}$, the digest was used directly for polymerase chain reaction (PCR).

\section{Design of Primers}

Primers specific for EGFR exons 18, 19, 20, 21, and for HER2 exons 19 and 20 were designed with the use of Primer Designer Software (Scientific and Education Software, Durham, NC, USA). The integrity of the primer systems for their PCR and the amplicon size were confirmed by subjecting the amplicons resulting from PCR to polyacrylamide gel electrophoresis on nondenaturing $8.0 \%$ polyacrylamide gels. The primers used are listed in Table 1. The extended primer set for EGFR exon 19 was used when one case appeared by HRMAA to contain a mutation but the mutation was confirmed only during reverse DNA sequencing. This suggested that the mutation was at or near the $5^{\prime}$ end of exon 19 and not easily seen by forward DNA sequencing. To confirm a mutation by bidirectional DNA sequencing, the extended exon 19 primer set was utilized. With these primers, the abnormal melting curve for this case was confirmed to be due to a mutation by bidirectional direct DNA sequencing.

\section{PCR and High-Resolution Melting Curve Analysis}

These procedures have been described in detail. ${ }^{3}$ Briefly, PCR was performed in a capillary cuvette (total volume $20 \mu \mathrm{l}$ ) on a Light Cycler (Roche Diagnostics, Indianapolis, IN, USA). All reactions contained dUTP instead of dTTP. Prior to PCR the samples were incubated with $1 \mathrm{U}$ of uracil $\mathrm{N}$-glycosylase (Amperase, Applied Biosystems, 
Table 1 Primers $^{\mathrm{a}}$

\begin{tabular}{|c|c|}
\hline & Sequence \\
\hline \multicolumn{2}{|l|}{ EGFR exon } \\
\hline \multicolumn{2}{|c|}{ Exon 18: (186 bp $\left.p^{\mathrm{b}}\right)$} \\
\hline Forward & 5'-CTGAGGTGACCCTTGTCTCTGTGTTCTT-3' \\
\hline Reverse & 5'-AGAGGCCTGTGCCAGGGACCTTA-3' \\
\hline \multicolumn{2}{|c|}{ Exon 19: (204 bp) } \\
\hline Forward & 5'-GCATGTGGCACCATCTCACAA-3' \\
\hline Reverse & 5'-CCTGAGGTTCAGAGCCATGGA-3' \\
\hline \multicolumn{2}{|c|}{ Exon 19 (extended, $259 \mathrm{bp}$ ) } \\
\hline Forward & 5'-TGGTAACATCCACCCAGATCAC-3' \\
\hline Reverse & $5^{\prime}$-CAGCTGCCAGACATGAGAAAAG-3' \\
\hline \multicolumn{2}{|c|}{ Exon 20: (248 bp) } \\
\hline Forward & $5^{\prime}$-CCATGCGAAGCCACACTGA-3' \\
\hline Reverse & 5'-CGTATCTCCCTTCCCTGATTACC-3' \\
\hline \multicolumn{2}{|c|}{ Exon 21: (236 bp) } \\
\hline Forward & 5'-GCAGAGCTTCTTCCCATGATGA-3' \\
\hline Reverse & 5'-GCTGACCTAAAGCCACCTCCT-3' \\
\hline \multicolumn{2}{|l|}{ HER2 exon } \\
\hline \multicolumn{2}{|c|}{ Exon 19: (214 bp) } \\
\hline Forward & 5'-CCACGCTCTTCTCACTCATATCC-3' \\
\hline Reverse & 5'-AAGAGAGACCAGAGCCCAGAC- $3^{\prime}$ \\
\hline \multicolumn{2}{|c|}{ Exon 20: (252 bp) } \\
\hline Forward & 5'-GGGTGTGTGGTCTCCCATAC-3' \\
\hline Reverse & $5^{\prime}$-GCAAAGAGCCCAGGTGCATA-3' \\
\hline
\end{tabular}

${ }^{\mathrm{a}}$ Primers were designed as described in Materials and methods.

${ }^{\mathrm{b}}$ Base pairs.

Foster City, CA, USA) to prevent 'carry-over' contamination. All samples contain the double stranded DNA-binding dye, LCGreen (Idaho Technology, Salt Lake City, UT, USA). After an initial preincubation step at $95^{\circ} \mathrm{C}$ (to denature the uracil glycosylase and activate the FastStart Taq DNA polymerase), cycling conditions consisted of 45 cycles, each cycle with a denaturation step at $95^{\circ} \mathrm{C}$ for $10 \mathrm{~s}$, followed by an annealing step at $60^{\circ} \mathrm{C}$ for $10 \mathrm{~s}$, and finally an extension step at $74^{\circ} \mathrm{C}$ for $0 \mathrm{~s}$. Transition rates were $10^{\circ} \mathrm{C} / \mathrm{s}$ from denaturation to annealing, $1^{\circ} \mathrm{C} / \mathrm{s}$ from annealing to extension, and $20^{\circ} \mathrm{C} / \mathrm{s}$ from extension to denaturation. After PCR, the samples were momentarily heated to $95^{\circ} \mathrm{C}$ and then cooled to $40^{\circ} \mathrm{C}$. An appropriate Light Cycler cuvette containing the sample of interest was transferred to the HR1, a high-resolution DNA melting analysis instrument (Idaho Technology, Salt Lake City, UT, USA) and a melting analysis was performed as described. ${ }^{3}$ Genomic DNA was used as a control. All samples were run in duplicate.

As discussed in the Results section, a common polymorphism is present in EGFR exon 20 at nucleotide position 2361. This is a change from $\mathrm{G}$ to $\mathrm{A}$ but it does not change the glutamine located at amino-acid residue position 787 . The wild type (CAG/CAG) has the highest melting temperature $\left(T_{\mathrm{m}}\right)$. The homozygous polymorphism (CAA/CAA) melts slightly below. The heterozygous (CAA/CAG) melts with the lowest $T_{\mathrm{m}}$. Therefore, cases in which the melting curve coincides with the homozygous wild type (showing the highest $T_{\mathrm{m}}$ ), can be concluded to contain only wild-type sequences without evidence of an activating mutation. All other cases require direct DNA sequencing because it is not possible to distinguish a potential activating mutation from the polymorphism in the homozygous or heterozygous state. The control for EGFR exon 20 requires genomic DNA containing the homozygous wild type which we obtained from a normal lymph node.

\section{DNA Sequence Analysis}

To correlate melting curve abnormalities with the presence or absence of a mutation, bidirectional DNA sequencing was performed on all cases and carrried out at the DNA sequencing core facility at the University of Utah. Analysis of the sequence was performed with the use of DNA sequencher 4.1.4 software from Gene Codes Corporation (Ann Arbor, MI, USA).

\section{Results}

\section{Patient Characteristics}

In all, 24 cases of squamous cell carcinoma affecting the head and neck area were retrieved from the surgical pathology files at the University of Utah Health Sciences Center. Since this was a first attempt to detect EGFR- or HER2-activating mutations by HRMAA in tumors from this area, all squamous cell carcinomas, including those tumors originating primarily from squamous mucosa of the oral cavity, sinuses and larynx as well metastatic squamous cell carcinomas from overlying skin were included. Five cases were primary cutaneous tumors which metastasized to or directly invaded the head and neck area, four cases were primary oral cavity tumors, eight cases were primary laryngeal tumors, four cases were primary tongue tumors, two cases originated from the maxillary sinus, and one case was primary in the nasal septum. There were 22 men and two women. Their ages ranged from 42 to 87 years with an average of $67.4 \pm 11.7$ years.

\section{High-Resolution Melting Amplicon Analysis}

HRMAA was performed to detect EGFR- and HER2activating mutations. Two of the 24 cases (8\%) showed melting curve abnormalities which were subsequently confirmed to be activating mutations by direct DNA sequencing. One case contained an EGFR exon 19-activating mutation (G729E) and the other contained an exon 20-activating mutation (N771YinsG). The corresponding melting curves obtained from HRMAA of these tumors are shown 

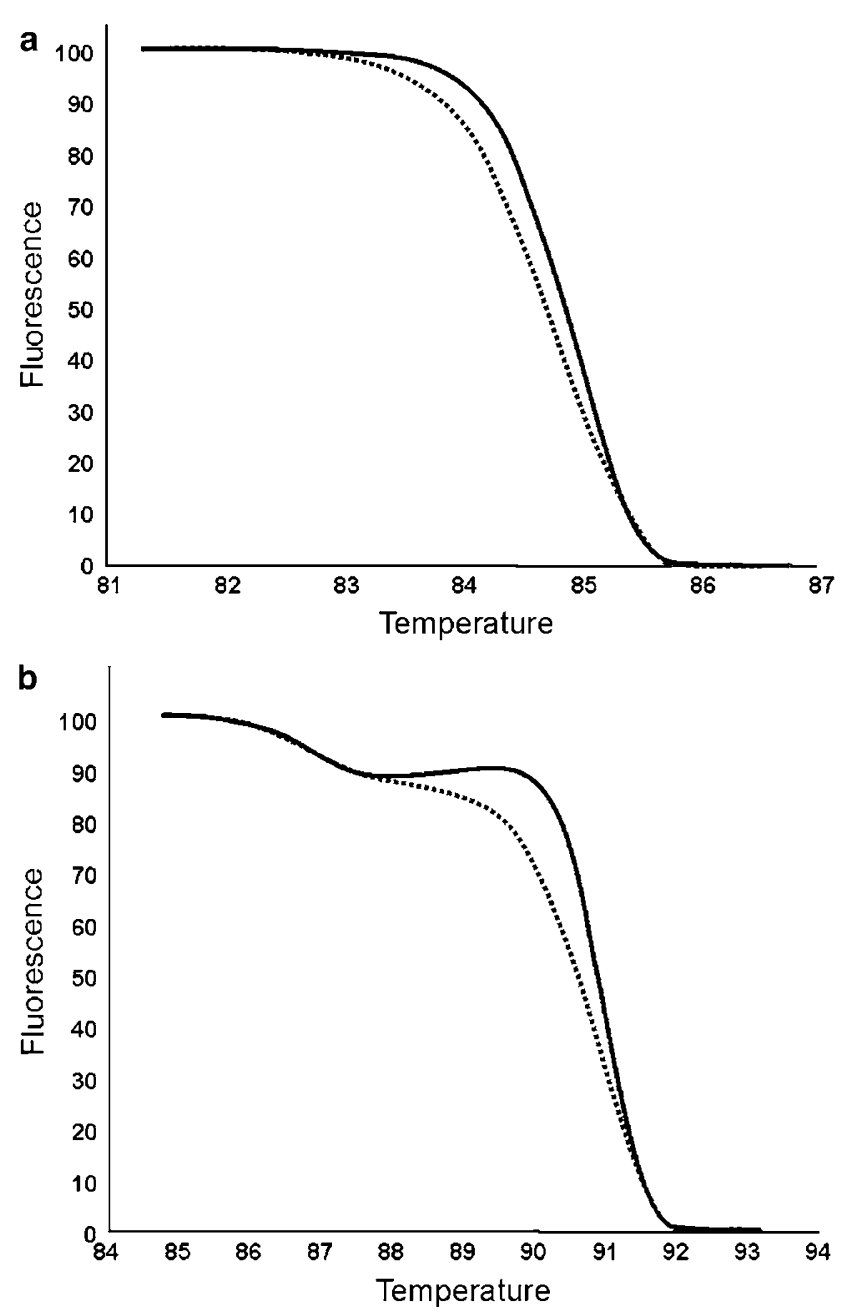

Figure 1 High-resolution melting amplicon analysis in squamous cell carcinoma. In all, 24 cases of squamous cell carcinoma affecting the head and neck were screened for EGFR- and HER2activating mutations as described in Materials and methods. All cases were followed up by direct DNA sequencing. Two of the 24 cases contained activating mutations. (a) HRMAA showing an EGFR-activating mutation in exon 19. The melting curve from the tumor (dotted line) is distinctly different than normal (solid line). DNA sequencing revealed an EGFR exon 19 mutation (G729E). (b) HRMAA showing an EGFR-activating mutation in exon 20. The melting curve from the tumor (dotted line) is distinctly different than normal (solid line). DNA sequencing revealed an EGFR exon 20 mutation (N771YinsG).

in Figure 1. No HER2-activating mutations were detected. These two presumptive activating mutations in EGFR have not been described before. Normal tissue from both cases was also analyzed for these mutations and the mutations were not present (data not shown). This suggests that the EGFR exon 19 G729E and EGFR exon 20 N771Yins771G represent newly described somatic mutations in the tumor. The histology of the EGFR mutation-positive cases does not show any distinguishing features as indicated shown in Figure 2. Both were interpreted as moderately differentiated squamous cell carcinomas. No HER2-activating mutations were found.

\section{Detection of Silent Mutations}

A common polymorphism is located in EGFR exon 20 at nucleotide position $2361 .^{5}$ This change is a G to A but it does not change the glutamine located at amino-acid residue position 787 . We observed this polymorphism in 17 of our 24 cases (71\%). The high prevalence of this silent mutation complicates HRMAA for EGFR exon 20. As discussed in Materials and methods, this necessitates that the HRMAA control for EGFR exon 20 be the homozygous wild type (highest $T_{\mathrm{m}}$ ) and that any melting curve that differs from this will require direct DNA sequencing. We also observed a silent mutation at nucleotide position 2578 in exon 21. This is a change of $\mathrm{C}$ to $\mathrm{T}$ which does not change the amino-acid arginine located at amino-acid residue 836. This mutation was detected in two of the 24 cases $(8 \%)$. One of the 24 cases $(4 \%)$ contained a polymorphism in the intron sequence immediately adjacent to the $5^{\prime}$ end of exon 18. This is a change of $\mathrm{C}$ to $\mathrm{T}$ which is located $8 \mathrm{bp}$ downstream from the start of exon 18. Our EGFR exon 18 primers amplify this small portion of the adjacent intron so this polymorphism is detected. No other EGFR polymorphisms were detected. No HER2 silent mutations were detected. The EGFR silent mutations are summarized in Table 2.

\section{Accuracy of HRMAA in Head and Neck Squamous Cell Carcinoma}

All cases which showed a normal melting pattern by HRMAA were found to contain a normal DNA sequence by follow-up direct DNA sequencing. All cases, in which the melting pattern was abnormal, were found to contain either activating mutations or silent mutations. No false positives or negatives were encountered. All curves were easily interpretable by visual inspection. This suggests that HRMAA could easily be used to screen for potential EGFR-and HER2-activating mutations in head and neck squamous cell carcinoma.

\section{Expression of EGFR in Head and Neck Squamous Carcinoma}

Overexpression of EGFR is common in squamous cell carcinoma of the head and neck. ${ }^{13}$ To determine the expression of EGFR in our series, all cases were analyzed by IHC. Of the 24 cases, two (8\%) were negative, one $(4 \%)$ showed $1+$ immunostaining, eight $(33 \%)$ showed $2+$ immunostaining, and 13 $(54 \%)$ showed $3+$ immunostaining. Both EGFR mutation-positive cases were interpreted as showing $3+$ EGFR expression as shown in Figure 2.

\section{Discussion}

Squamous cell carcinoma of the head and neck is one tumor which is known to overexpress EGFR. 



Figure 2 Histology and EGFR immunostaining in squamous cell carcinoma with EGFR-activating mutations. The two cases as described in Figure 1 with EGFR-activating mutations were screened for EGFR expression by immunohistochemistry. The stains were interpreted on a 0 to $3+$ scale as described in Materials and methods. (a) $\mathrm{H}$ and E-stained section of tumor with exon 19 mutations ( $\times 250)$. (b) $\mathrm{H}$ and E-stained section of tumor with exon 20 mutation $(\times 250)$. (c) EGFR IHC in the tumor with an exon 19 mutation. The stain was interpreted as $3+(\times 250)$ (d) EGFR IHC in the tumor with an exon 20 mutation. The stain was interpreted as $3+(\times 150)$.

Table 2 Silent EGFR mutations in squamous cell carcinoma $(n=24)$

\begin{tabular}{lccc}
\hline Location & Exon 20 & Exon 21 & Intron 17 \\
\hline $\begin{array}{l}\text { Nucleotide } \\
\text { position }\end{array}$ & 2361 & 2578 & $\begin{array}{c}-8 \text { bp from start } \\
\text { of exon 18 }\end{array}$ \\
$\begin{array}{l}\text { Change } \\
\begin{array}{l}\text { Number and } \\
\text { percent of affected } \\
\text { cases }\end{array}\end{array}$ & $\mathrm{G} \rightarrow \mathrm{A}$ & $\mathrm{C} \rightarrow \mathrm{T}$ & $\mathrm{C} \rightarrow \mathrm{T}$ \\
& & $2(81 \%)$ & $1(4 \%)$ \\
\hline
\end{tabular}

This observation is the basis for therapeutic protocols attempting to inhibit the action of this receptor either by monoclonal antibodies ${ }^{14}$ or small molecule tyrosine kinase receptors. ${ }^{9}$ Recent data in non-smallcell lung cancer, another tumor known to over- express EGFR, suggests that the response to the active site EGFR inhibitors, gefitinib and erlotinib, is correlated with an underlying EGFR-activating mutation in the tumor. ${ }^{5} \mathrm{~A}$ recent report describes the presence of EGFR-activating mutations in a small subset of patients with head and neck squamous cell carcinoma ${ }^{11}$ which suggests that these patients might also benefit from gefitinib or erlotinib therapy. No studies have yet correlated the response of head and neck squamous cancers to EGFR inhibitors with underlying EGFR-activating mutations in the tumor.

We recently used the technique of HRMAA to screen for activating mutations in GISTs $^{15}$ and melanoma. ${ }^{3}$ HRMAA is a newly described method which can be used for mutation scanning. If a tumor has a mutation in an exon of interest, after DNA isolation and PCR amplification, the PCR products 
will contain a mixture of DNA molecules; some with the mutation (from the tumor) and some without (from normal cell contamination and from heterozygous tumor cells). Denaturation and reannealing of the PCR products will yield a mixture of homoduplexes (mutant/mutant and normal/normal) and heteroduplexes (mutant/normal). As a result of the base pair mismatch in the heteroduplexes, these molecules will have a lower $T_{\mathrm{m}}$ than normal. Their contribution to the melting curve analysis performed on the PCR-amplified products is easily detected with high-resolution technology. ${ }^{16}$

In this study, we used HRMAA to screen cases of squamous cell carcinoma involving the head and neck for both EGFR- and HER2-activating mutations. We included primary cutaneous squamous carcinomas, which only secondarily involved the head and neck area for two reasons. First, there is little information on EGFR-activating mutations in squamous carcinoma, and second, a recent article describes the marked response of a cutaneous squamous cell carcinoma involving the head and neck to gefitinib. ${ }^{17}$ Remarkably, one of our primary cutaneous squamous cancers contained an EGFR exon 19-activating mutation. This tumor originated on the chin, was deeply invasive requiring a partial resection of the mandible, and was also metastatic to the lung. This result suggests that perhaps EGFRactivating mutations might characterize a group of aggressive squamous cell carcinomas of the skin and that these tumors might respond to gefitinib or erlotinib. The other EGFR activating mutation discovered was in exon 20 (N771YinsG). The tumor with this mutation was a squamous cell carcinoma originating from the maxillary sinus. No mutations were found in HER2.

All cases screened with HRMAA were followed up by direct DNA sequencing. Every case in which the PCR products showed a normal melting curve was found to contain a normal DNA sequence and every case in which the PCR products showed an abnormal melting curve was found either to contain a silent or activating mutation. This remarkable sensitivity and specificity has been observed previously. ${ }^{3,15}$ In the absence of a large deletion or duplication, is not possible, however, to accurately distinguish silent mutations from activating mutations. In our laboratory, all abnormal melting curves still undergo direct DNA sequencing. However, it appears from our data and well as that from others, ${ }^{11}$ that the frequency of EGFR mutations in squamous cell carcinoma will be low which suggests that most cases can be easily eliminated as having potential activating mutations simply by scanning with HRMAA. Attention can then be directed to cases showing abnormal melting curves.

Drugs specifically targeted against EGFR or HER2 represent an important area of basic and clinical research. The activity of these drugs against human malignancies will need to be determined in large clinical trials. Recent articles have emphasized the importance of correlating molecular data with clinical response in these trials. ${ }^{18,19}$ HRMAA may prove useful in providing some of this molecular data.

\section{Acknowledgements}

We thank Trena Held for preparing the tables. We are grateful to the Associated and Regional Pathologists (ARUP) Institute for Clinical and Experimental Pathology for support of this work. We thank Dr Carl Wittwer for his help and advice with DNA melting analysis.

\section{References}

1 Dibb NJ, Dilworth SM, Mol CD. Switching on kinases: oncogenic activation of BRAF and the PDGFR family. Nat Rev 2004;4:718-727.

2 Corless CL, Fletcher JA, Heinrich MC. Biology of gastrointestinal stromal tumors. J Clin Oncol 2004;22: 3813-3825.

3 Willmore-Payne C, Holden JA, Tripp S, et al. Human malignant melanoma: detection of BRAF- and c-kitactivating mutations by high-resolution amplicon melting analysis. Hum Pathol 2005;36:486-493.

4 Xing M. BRAF mutation in thyroid cancer. Endocr Relat Cancer 2005;12:2452-2462.

5 Takano T, Ohe Y, Sakamoto H, et al. Epidermal growth factor receptor gene mutations and increased copy numbers predict gefitinib sensitivity in patients with recurrent non-small-cell lung cancer. J Clin Oncol 2005;23:6829-6837.

6 Stephens P, Hunter C, Bignell G, et al. Lung cancer: intragenic ERBB2 kinase mutations in tumours. Nature 2004;431:525-526.

7 Pao W, Miller VA. Epidermal growth factor receptor mutations, small-molecule kinase inhibitors, and nonsmall-cell lung cancer: current knowledge and future directions. J Clin Oncol 2005;23:2556-2568.

8 Vermorken JB. Medical treatment in head and neck cancer. Ann Oncol Suppl 2005;2:ii258-ii264.

9 Cohen EE, Rosen F, Stadler WM, et al. Phase II trial of ZD1839 in recurrent or metastatic squamous cell carcinoma of the head and neck. J Clin Oncol 2003;21: 1980-1987.

10 Wirth LJ, Haddad RI, Lindeman NI, et al. Phase I Study of Gefitinib Plus Celecoxib in recurrent or metastatic squamous cell carcinoma of the head and neck. Clin Oncol 2005;23:6976-6981.

11 Lee JW, Soung YH, Kim SY, et al. Somatic mutations of EGFR gene in squamous cell carcinoma of the head and neck. Clin Cancer Res 2005;11:2879-2882.

12 Tripp SR, Willmore-Payne C, Layfield LJ. Relationship between EGFR overexpression and gene amplification status in central nervous system gliomas. Anal Quant Cytol Histol 2005;27:71-78.

13 Pomerantz RG, Grandis JR. The epidermal growth factor receptor signaling network in head and neck carcinogenesis and implications for targeted therapy. Semin Oncol 2004;31:734-743.

14 Burtness B. The role of cetuximab in the treatment of squamous cell cancer of the head and neck. Expert Opin Biol Ther 2005;5:1085-1093. 
15 Willmore C, Holden JA, Zhou L, et al. Detection of c-kit-activating mutations in gastrointestinal stromal tumors by high resolution amplicon melting analysis. Am J Clin Pathol 2004;122:206-216.

16 Zhou L, Wang L, Palais R, et al. High-resolution DNA melting analysis for simultaneous mutation scanning and genotyping in solution. Clin Chem 2005;51: 1770-1777.

17 Baltaci M, Fritsch P, Weber F, et al. Treatment with gefitinib (ZD 1839) in a patient with advanced cutaneous squamous cell carcinoma. Br J Dermatol 2005;153:234-236.

18 Johnson BE, Janne PA. Selecting patients for epidermal growth factor receptor inhibitor treatment: a FISH story or a tale of mutations? J Clin Oncol 2005;23:6813-6816.

19 Siegel-Lakhai WS, Beijnen JH, Schellens JH. Current knowledge and future directions of the selective epidermal growth factor receptor inhibitors erlotinib (tarceva(r)) and gefitinib (iressa(r)). Oncologist 2005; 10:579-589. 